\title{
Metachronous multicentric recurrent giant cell tumour of metacarpals: a case report
}

\begin{abstract}
Giant Cell Tumour (GCT) of bone usually presents around knee and distal radius in 20 to 40 years age group. We present a case that developed in first metacarpal in a lady of 57 years which also recurred after initial treatment and involves second metacarpal.

In November 2015 she presented in our institution with recurrence of GCT of the 1st MC of left hand. Initial presentation was intraosseous Capancci grade III lesion. That was treated with narrow margin resection, interposition bone cement on a Kirschner wire (K wire) which started recurring after 8 months. But presented to us after another one year when extensive extension within soft tissue took place. MRI showed second metacarpal bone and extensive soft tissue involvement. Lung metastasis was excluded.

Under tourniquet control the mass along with bone cement was removed, thoroughly cleaned and tricortical bone graft from iliac crest was placed in position with $4 \mathrm{~K}$-wires. After closure of wound hand and forearm was placed in plaster of Paris slab. Stitches were removed after 2 weeks but cast continued for 2 months and $\mathrm{K}$ wires continued for 3 months when graft ends started uniting and partially incorporated. Histopathology reported as GCT in both sites without malignancy. Movements of carpometacarpal and metacarpophalangeal joints were restricted but interphalangeal joint exhibit $50 \%$ motion. Wrist became free. In 1 year 3 months follow up there is no evidence of recurrence or metastasis. She is on bisphosphonate and vitamin D therapy.
\end{abstract}

Keywords: GCT, Metacarpal, Recurrence, Multicentric, Metachronous, Tricortical Bone Graft
Volume 10 Issue I - 2018

\author{
Jitendra Nath Pal,' Amiya Kumar Bera, ${ }^{2}$ \\ Amarendra Nath Roy2 \\ 'Professor, Department of Orthopedics, University of Health \\ Sciences, India \\ ${ }^{2}$ Assistant Professor, Department of Orthopedics, University of \\ Health Sciences, India
}

\begin{abstract}
Correspondence: Jitendra Nath Pal, Professor, Department of Orthopedics, Murshidabad Medical College, West Bengal, India, F-505, Maitri Apartment, 255, N S C Bose Road, Kolkata, West Bengal, India, Tel 9133 248I650I, Fax 91332481650, Email jitendranathpal@gmail.com
\end{abstract}

Received: August 15, 2017| Published: January 10, 2018
Abbreviations: GCT, Giant Cell Tumour; MC, Metacarpal; $\mathrm{K}$ wire, Kirschner wire; MRI, Magnetic Resonance Imaging; HP, Histopathology

\section{Introduction}

Giant Cell Tumour (GCT) of bone mostly develops around Knees and distal radius in 20 to 40 years age group with little higher incidence in female. ${ }^{1-4}$ Occurring in small bones like Metacarpal (MC) is rare multicentric involvement is rarest $(1 \%) .{ }^{5,6}$ When multicentric it may be synchronous or metachronous lesion. General agreement of treatment in earlier presentation is intralesional excision and filling up of the cavity with bone, bone substitutes, bone cement. ${ }^{7}$ Rarely resection and reconstructions are done. Recurrence of GCT do occur in $25 \% .{ }^{4}$ In this study we are presenting a case of metachronous multicentric GCT of metacarpals in a lady of 60 years which recur after initial treatment and tricortical cancellous strut graft is used during treatment of recurrence.

\section{Case report}

In November 2015 one 60 years lady presented in a newly recognised Medical College of eastern India (Murshidabad Medical College) with recurrence of GCT of the 1st MC of left hand and soft tissue involvement. One year back she was presented in another medical college with intraosseous Capancci grade III lesion of the same site of two years duration where it was treated with narrow margin resection, interposition bone cement on a Kirschner wire (K wire) (Figure 1). It started recurring after 8 months of resection. But presented to us after another one year when extensive extension within soft tissue took place. In plain $\mathrm{x}$ ray one small osteolytic lesion was noticed over mid shaft of second metacarpal of the same hand which was not seen in earlier radiographs. MRI was done to identify adjacent bone involvement and to know extent of the lesion in soft tissue (Figure 2). Involvement of regional lymph node and lung metastasis were excluded clinically and radiologically.
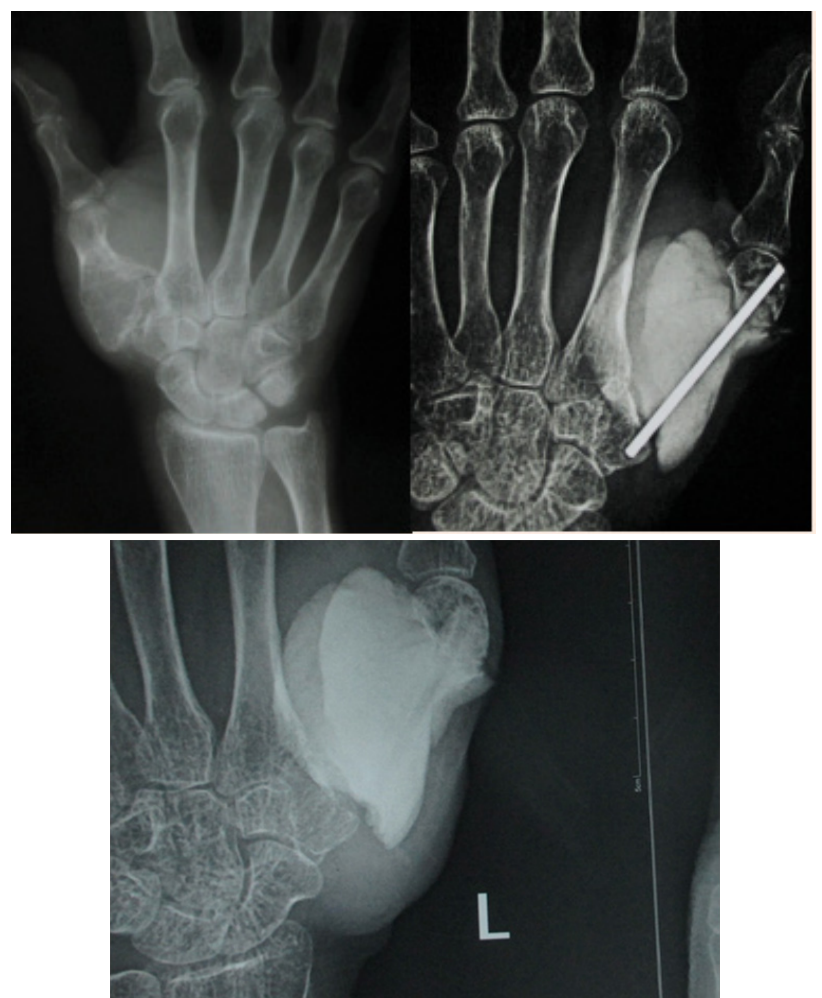

Figure I a. Initial X-ray initial presentation 3 years back; post-operative $\mathrm{X}$-ray after initial operation; b. immediate; c. subsequent. 

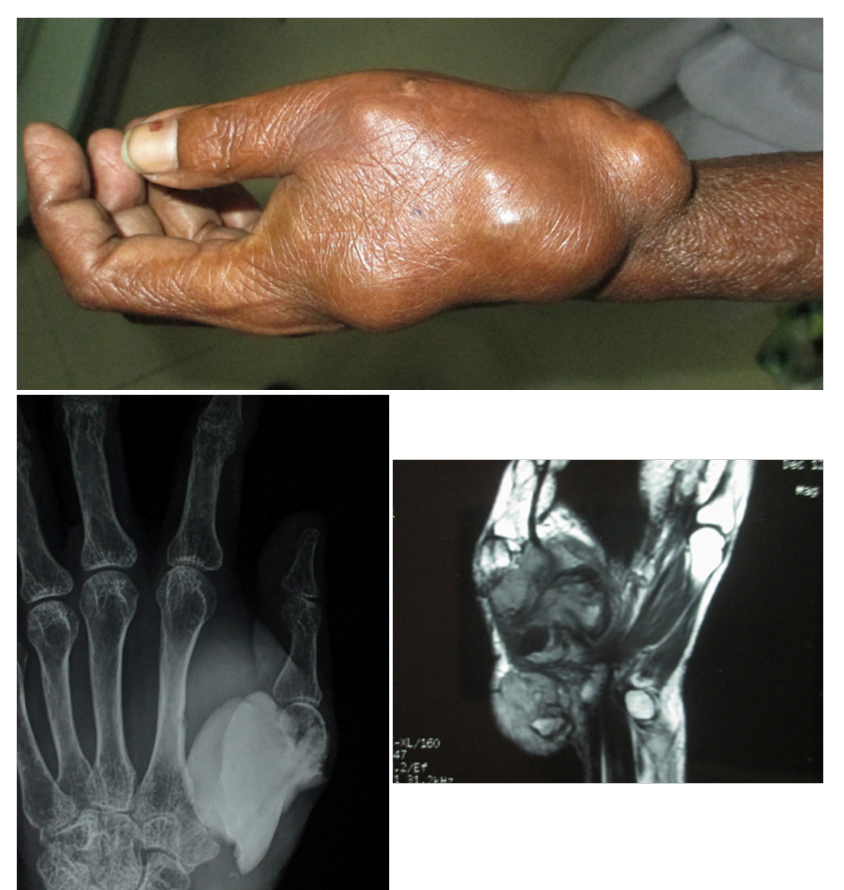

Figure 2 a. Clinical picture on presentation one year after recurrence; $b$. $X$-ray at that time showing soft tissue and second MC involvement; c \& d. MRI show similar findings but without involvement of major nerve and vessels.

\section{Technique and result}

Under tourniquet control and regional anaesthesia through dorsoradial incision the mass along with bone cement was removed, meticulously cleaning of original tumour site was done. Excised tissue was preserve for histopathology (HP). With a separate set of clean instrument the osteolytic area second metacarpal was curetted and the material is preserved in separate jar for HP study. Tricortical bone graft from iliac crest of appropriate size was harvested and placed in position with $4 \mathrm{~K}$-wires. After closure of wound hand and forearm was placed in plaster of Paris slab. Stitches were removed after 2 weeks but cast continued for 2 months and $\mathrm{K}$ wires continued for 3 months when graft ends started uniting and partially incorporated (Figure 3). Histopathology reported as GCT without malignancy from both the specimens. $75 \%$ movements of carpometacarpal and metacarpo-phalangeal joints were restricted but interphalangeal joint exhibit 50\% motion (Figure 4). Wrist became free. In 1 year 3 months follow up there is no evidence of recurrence or metastasis. Considerable amount of reactive consolidating bone formation around $2^{\text {nd }} \mathrm{MC}$ radiologically.

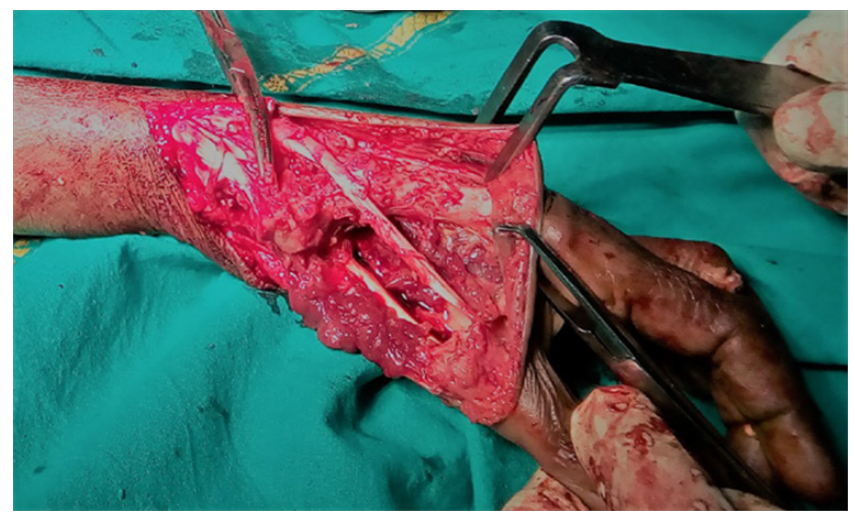

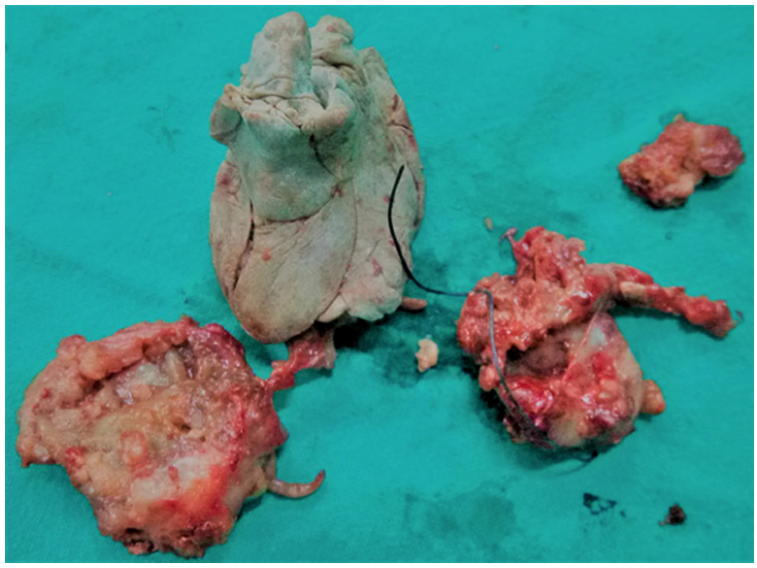

Figure 3 Peroperative pictures show a. Involvement of second MC; b. Removed materials.
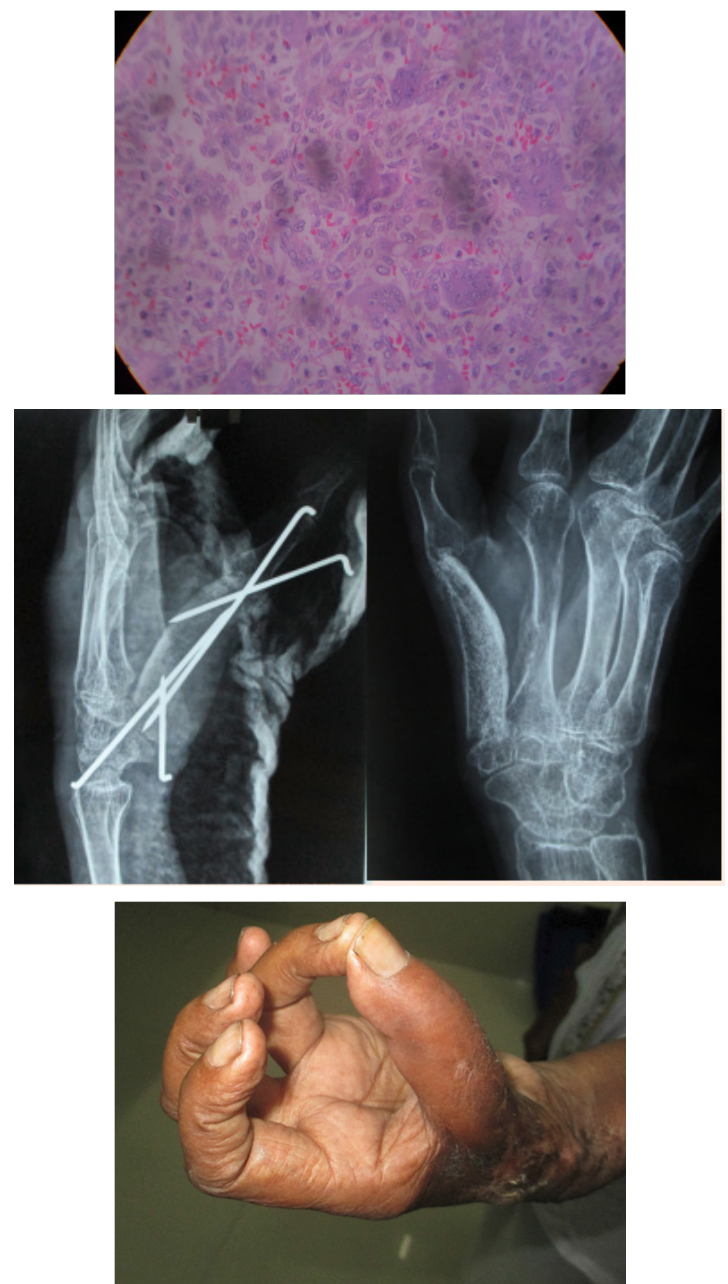

Figure 4 a. Micro-photographic picture of histopathology, follow up $X$ Rays show; b. immediate post-operative; c. after incorporation of grafted bone matured reactive bone around second MC without evidence of recurrence; d. clinical result.

\section{Discussion}

Hoch et al. ${ }^{6}$ classified multicentric GCT as being "synchronous" when multiple tumours had been discovered at the initial presentation or when a second tumour had been diagnosed within six months after the first. If the second tumour developed more than six months after the 
first lesion, the lesions were considered to be "metachronous". A germline genetic abnormality that predisposes patients to develop multiple tumours has also been postulated but no familial cases have been reported so far. ${ }^{6}$ We designed the second lesion as metachronous as it started more than 2 years after the primary lesion. Various pathogenic mechanisms of multicentricity have been suggested including contiguous spread, iatrogenic tumour cell seeding, benign metastasis, malignant transformation and de novo multifocal formation. ${ }^{6,8}$ In the present study it is likely to be contiguous spread. Balke et al. ${ }^{9}$ stated histological characteristics of multricentric GCT do not differ from that of solitary tumours although some cases may contain fibroblastic and fibrohistiocytic areas which are probably features of degeneration or involution ${ }^{9}$ Histological picture of subsequent lesion is same as that of the parent lesion and do not demonstrate fibroblastic or fibrohistocytic differentiation.

Reiner et al. ${ }^{10}$ reported incidences of pulmonary metastasis is $5-10 \%$ in patients with multicentric GCTs as against $1-2 \%$ in patients with solitary GCTs. ${ }^{10}$ Whereas Hoch et al. \& Belk et al. ${ }^{6}{ }^{10}$ find it rarely and none in their series respectively. In the present case at 1.5 years there is no pulmonary metastasis. It is understandable that the follow up period is too short to comment.

Tse et al. ${ }^{11}$ stated local recurrence rate was $4.2 \%$ in patients treated with bisphosphonate and 30\% in a control group. ${ }^{11}$ The case under investigation is receiving bisphosphonate and Vitamin D as adjuvant therapy. Denosumab is more effective adjuvant therapy against GCT of bone but cannot be administered to the present case because of the cost. $^{12}$

Although meticulous curettage and autologous cancellous bone graft or marginal margin resection and reconstruction with strut bone graft are well established methods of treatment of GCT of small bones, possibly to reduce recurrence, the concerned surgeon planned for two stage procedure with bone cement implanting after resection as stage one and if there is no recurrence in one year or so appropriate reconstruction as the second stage. Where there is indication of strut bone graft Author's preference is using tricortical iliac crest rather than cortical shaft of fibula. Because incorporation and union is faster in cancellous bone and confer reasonable strength also. ${ }^{14}$ In this case it is taken up within 3 months.

\section{Acknowledgements}

Dr. Arya Sen, Department of Pathology, Murshidabad Medical College, is acknowledged for his contribution in collecting microphotography and its interpretations.

\section{Conflicts of interest}

Authors declare no conflict of interest in conducting this study.

\section{Patient consent form}

Duly signed informed consent is obtained from the patient while conducting this study.

\section{References}

1. Derbel O, Zrounba P, Chassagne Clément C, et al. An unusual giant cell tumor of the thyroid: case report and review of the literature. $J$ Clin Endocrinol Metab. 2013;98:1-6.

2. Raskin KA, Schwab JH, Mankin HJ, et al. Giant cell tumor of bone. $J$ Am Acad Orthop Surg. 2013;21:118-126.

3. Chakarun CJ, Forrester DM, Gottsegen CJ, et al. Giant cell tumor of bone: review, mimics, and new developments in treatment. Radiographics. 2013;33(1):197-211

4. Fletcher CDM, Unni KK, Mertens F. World Health Organisation Classification of Tumours: Pathology and Genetics of Tumours of Soft Tissue and Bone. Lyon, IARC Press, France, 2002;pp.1-415.

5. Averill RM, Smith RJ, Campbell CJ. Giant-cell tumours of the bones of the hand. Journal of Hand Surg. 1980;5:39-50.

6. Hoch B, Inwards C, Sundaram M, et al. Multicentric giant cell tumor of bone: clinicopathologic analysis of thirty cases. The Journal of Bone and Joint Surgery Series A. 2006;88(9):1998-2008.

7. Carrasco CH, Murray JA. Giant cell tumours. Orthop Clin North Am 1989;20(3):395-405.

8. Dhillon Mandeep S, Prasad Prabhudev. Multicentric giant cell tumour of bone. Acta Orthop Belg. 2007;73(3):289-299.

9. Balke M, Henrichs MP, Goshegeretal G. Giant cell tumors of the axial skeleton. Sarcoma. 2012:10.

10. Reiner W, Frank B, Dirk L, et al. Multicentric Giant Cell Tumor of Bone: Synchronous and Metachronous Presentation. Case Reports in. Orthopedics. 2013:5.

11. Tse LF, Wong KC, Kumta SM, et al. Bisphosphonates reduce local recurrence in extremity giant cell tumor of bone: a case-control study. Bone. 2008;42(1):68-73.

12. Branstetter DG, Nelson SD, Manivel JC, et al. Denosumab induces tumor reduction and bone formation in patients with giant-cell tumor of bone. Clin Cancer Res. 2012;18(16):4415-4424.

13. Jitendra Nath Pal, Anindya Basu, Sunit Hazra, et al. Bizarre Parosteal Osteochondral Proliferation of Metatarsal is reconstructed by Free Tricortical Iliac Bone Graft: A Case Report JEBMH. 2014;1(17):2197-2203. 